\title{
Identificando exportaciones dominicanas sensibles al Tratado Trans-Pacífico (TTP)
}

\author{
Por Pável Isa Contreras*
}

Recibido: marzo/2017 - Aceptado: julio/2017

\section{Resumen}

El Tratado Trans-Pacifico (TTP), un propuesto mega-acuerdo económico entre 12 economías del mundo, siete de Asia y cinco de América, tendría el potencial de desplazar del mercado estadounidense las exportaciones originarias de terceros países, como la República Dominicana. Este análisis identifica las exportaciones dominicanas hacia Estados Unidos que, por su importancia para el pais, por las Ventajas Comparativas Reveladas de esos productos de países socios de Estados Unidos en el TTP, y por las barreras arancelarias que serían desmontadas como resultado del acuerdo, podrían ser sensibles al mismo. Se concluye que un total de 13 partidas arancelarias podrían ser afectadas y cinco países podrían ser beneficiarios. No obstante, casi la totalidad del efecto desplazamiento se concentra en prendas de vestir y Vietnam sería el gran beneficiario. El efecto específico sobre las exportaciones dominicanas podría ser muy pequeño porque el efecto global también lo es y se distribuiría entre un gran número de paises suplidores.

Palabras clave: TTP; exportaciones; ventajas comparativas reveladas; aranceles.

Códigos del JEL: F1, F4, F6

* Escuela de Economía y Negocios, Instituto Tecnológico de Santo Domingo (INTEC) y Observatorio Dominicano de Comercio Internacional (ODCI). Dirección: Avenida de Los Próceres \#49, Los Jardines del Norte 10602, Santo Domingo, República Dominicana, Apartado postal 342-9 y 249-2. T: 809-567-9271 - Fax: 809-566-3200 (e-mail: pavel.isa@intec. edu.do). Agradecimientos: El autor agradece a William Miguel Román por su apoyo como asistente de investigación. 


\title{
Identifying Dominican exports sensitive to the Trans-Pacific Partnership (TPP)
}

\author{
By Pável Isa Contreras*
}

Submitted: March 2017 - Accepted: July 2017

\begin{abstract}
The Trans-Pacific Partnership (TPP), a proposed economic mega-agreement between 12 economies, seven of Asia and five of Ameri$c a$, would have the potential to displace exports from third countries, such as the Dominican Republic, from the US market. This analysis identifies Dominican exports to the US, which, because of their importance to the country, the Revealed Comparative Advantages of those products in TPP partners of US, and the tariff barriers that would be dismantled as a result of the agreement, could be sensitive to the agreement. It is concluded that a total of 13 tariff headings could be affected and five countries could be beneficiaries. However, almost the entire displacement effect is concentrated in clothing and Vietnam would be the great beneficiary. The specific effect on Dominican exports could be very small because the overall effect is also small and would be distributed among a large number of supplier countries.
\end{abstract}

Keywords: TPP; globalization; revealed comparative advantages; tariffs; free trade.

JEL Codes: F1, F4, F6

* School of Economics and Business, Instituto Tecnológico de Santo Domingo (INTEC) and Observatorio Dominicano de Comercio Internacional (ODCI). Address: Avenida de Los Próceres \#49, Los Jardines del Norte 10602, Santo Domingo, Dominican Republic. P.O. Box 342-9 y 249-2. T: 809-567-9271•Fax: 809-566-3200 (e-mail: pavel.isa@intec.edu.do). Acknowledgements: The author thanks William Miguel Román for his support as research assistant. 


\section{Introducción}

En octubre de 2015, ministros de 12 países de América y Asia anunciaron el fin de las negociaciones de un ambicioso acuerdo que liberalizaría el comercio entre dichos países y les dotaría de un marco legal para sus relaciones económicas. El acuerdo ha sido denominado el Acuerdo de Asociación Trans-Pacífico o Tratado Trans-Pacífico (TTP).

El avance en las negociaciones hizo sonar las alarmas en Centroamérica y la República Dominicana porque, en la medida en que el acuerdo elimina barreras a las importaciones de Estados Unidos de mercancías originarias de los países socios, erosionaría las preferencias arancelarias que disfrutan los países de la región en el marco del tratado de libre comercio con Estados Unidos, conocido como DR-CAFTA. El resultado de esa erosión podría ser la desviación de comercio, la cual implicaría una reducción de las exportaciones de la región hacia Estados Unidos de mercancías que enfrenten una oferta competitiva en ese mercado de los demás países miembros del acuerdo.

Las exportaciones de la región hacia el mercado de Estados Unidos pueden verse afectadas si, además de los aranceles, las reglas de origen que se negociaron para el acceso de las mercancías a dicho mercado son más flexibles que las que se acordaron en el DR-CAFTA para las mercancías originarias de Centroamérica y la República Dominicana.

Adicionalmente, aunque en menor medida, las economías de la región pueden verse impactadas negativamente debido a la erosión del nivel de acceso relativo a los mercados de otros países miembros del TTP, como resultado del propio acuerdo, que otorgaría ventajas recíprocas. Por último, la región también podría ser afectada por el potencial desvío de inversiones que puede resultar del acuerdo en la medida en que debilita, en términos relativos, la posición competitiva 
de la región para atraerlas, tanto como resultado del debilitamiento de las preferencias relativas como de las reglas de vinculación económica entre los países miembros, tales como las asociadas a las inversiones, al tratamiento de la propiedad intelectual, a la facilitación de comercio y al tratamiento a las compras gubernamentales, entre otras.

Evaluar el potencial impacto del TTP en la República Dominicana implicaría medir los efectos en todas estas dimensiones. El propósito de este trabajo es, sin embargo, más acotado y se centra en la preocupación fundamental sobre el impacto potencial del acuerdo sobre las exportaciones hacia el mercado de Estados Unidos. En ese sentido, se pretende identificar las actividades de exportación que podrían ser más afectadas por el acuerdo y aproximarse a dimensionar su efecto.

En la segunda parte se hace una breve referencia a algunos de los estudios más importantes sobre las implicaciones del TTP para las economías envueltas. En la tercera, se describe el alcance del TTP desde los puntos de vista económico, comercial y temático. La cuarta parte presenta la discusión en torno a la estructura de las exportaciones de los países miembros del TTP hacia Estados Unidos por países, la importancia de estas para esas economías y los niveles de concentración del comercio que prevalecen. En la quinta parte se describe la metodología utilizada para identificar y dimensionar las amenazas. Finalmente, la sexta parte está dedicada a la explicación de los resultados del ejercicio de aproximación a las actividades más amenazadas y las posibles sensibilidades, además de proveer una síntesis de los mismos.

\section{Estudios sobre el TTP}

A raíz de las negociaciones a puerta cerrada del texto del TTP, un número de trabajos aparecieron procurando dar cuenta de las implicaciones económicas de un acuerdo de esa naturaleza. La mayor parte de ellos se realizó para Estados Unidos y sus resultados no estuvieron exentos de controversias. 
El trabajo más conocido de todos es el que elaboró la USITC (2016), en el cual se estimó los efectos a nivel global y en sectores específicos, utilizando un modelo de equilibrio general computable dinámico; el resultado arrojado fue que dichos efectos eran positivos, pero pequeños.

Otros dos estudios relevantes fueron el de Petri, Plummer \& Zhai (2012) y el realizado por Petri \& Plummer (2016), siendo el segundo una actualización del primero. Estos también encontraron efectos positivos para la economía estadounidense, la cual concentraría la mayor parte del beneficio absoluto. Pero, además, estimaron efectos positivos para el resto de los países, en particular para Japón, Malasia y Vietnam.

Por el contrario, un controvertido trabajo de Capaldo \& Izurieta (2016) encontró que los efectos en el producto eran menores a los estimados por otros estudios y halló efectos negativos, en particular, sobre la equidad y los empleos. Por su parte, Lawrence (2016) cuestiona sus hallazgos.

El Banco Mundial (2016) también publicó resultados sobre el impacto macroeconómico potencial del TTP, encontrando efectos positivos agregados sobre el producto y el comercio, aunque advierte sobre riesgos y discute políticas de respuesta.

Por su parte, el Peterson Institute auspició estudios sobre los potenciales impactos del TTP en una variedad de aspectos tales como: el mercado de trabajo y la distribución del ingreso (Lawrence \& Moran, 2016), los flujos de comercio (Freund, Moran \& Oliver, 2016), el comercio de textiles y las confecciones a la luz de las reglas de origen (Elliot, 2016) y el comercio de servicios (Hufbauer, 2016). Es relevante también mencionar el aporte de J. K. Jackson del Congressional Research Service de Estados Unidos, quien hace un balance de un importante número de estudios sobre los efectos del acuerdo (Jackson, 2016). 
Por último, Strutt, Minor \& Rae (2015) desarrollaron un análisis a partir de un modelo de equilibrio general computable dinámico para medir el impacto en Nueva Zelandia; mientras que Cheong \& Tongzon (2013) hicieron un análisis comparativo del TTP y del RCEP (Regional Comprehensive Economic Partnership), un acuerdo trans-asiático que incluye, además de los países del TTP de ese continente, a China, Camboya, Indonesia, Lao, Muanmar, Filipinas, Tailandia, India y Corea del Sur.

\section{El alcance del Tratado Trans-Pacífico (TTP)}

\subsection{Alcance geográfico y económico}

El Acuerdo de Asociación Trans-Pacífico o TTP tendría un alcance geográfico inigualable, vinculando territorios no solo a través del Océano Pacífico, sino cruzando hemisferios (Norte y Sur). Involucra 12 países, cinco de los cuales son de América (Canadá, Estados Unidos, México, Perú y Chile) y siete corresponden a Asia (Japón, Vietnam, Singapur, Brunei, Malasia, Australia y Nueva Zelandia).

Según datos del Banco Mundial, en 2014 el Producto Interno Bruto (PIB) agregado de todos los países del TTP alcanzó la suma de poco menos de US\$ 28 billones, lo que equivale a un 36\% del PIB mundial de ese año. Sin embargo, el valor total del producto está muy concentrado en unas pocas grandes economías, especialmente la de Estados Unidos, que explicó un $62.1 \%$ del total, y la de Japón, con un $16.4 \%$, para un total combinado de $78.5 \%$. La tercera economía más grande del bloque es la de Canadá, que apenas alcanzó representar el 6.4\% del total, menos del $40 \%$ de Japón y apenas un 10\% de Estados Unidos ${ }^{1}$.

En cuanto al peso del bloque en el comercio mundial, este mostró ser mucho más modesto. En 2014 su participación fue de 11.1\% y

\footnotetext{
${ }^{1}$ El Índice de Herfindhal de concentración del producto en ese grupo de países alcanzó 4,242; excluyendo a Estados Unidos se redujo hasta 2,531.
} 
desde inicios de la década pasada, cuando superaba el 16\%, ha mostrado una tendencia al declive, la cual probablemente se asocia a que el crecimiento del comercio mundial ha sido más acelerado en otros países del mundo, en particular en China.

La concentración del comercio también es menor que la del PIB. Estados Unidos, la economía más grande, explicó el 37\% de todas las exportaciones de los países del grupo, a pesar de explicar más del 61\% del PIB. Estas alcanzaron un total de US\$ 1.62 billones en 2014. Japón ocupó el segundo lugar, con US\$ 733 mil millones de exportaciones o el 16.7\% del total. Le siguió Canadá, con un 10.5\% (US\$ 462 mil millones), Singapur con el 9.3\% (US\$ 410 mil millones) y México con un $8.7 \%$ (US\$ 383 mil millones). Estos cinco países explicaron el $82 \%$ de las exportaciones del grupo, mientras los otros siete (Australia, Malasia, Vietnam, Chile, Perú, Nueva Zelandia y Brunei) justificaron el $18 \%$ restante.

$\mathrm{El}$ acuerdo contiene 30 capítulos. Estos incluyen temas tradicionales como el acceso a mercado (capítulo 2), las reglas de origen (capítulo 3), la facilitación de comercio y defensa comercial (capítulos 5 y 6), las medidas sanitarias y los obstáculos técnicos (capítulos 7 y 8), e inversiones y servicios (capítulos 9 y 10). También, incluyen los temas de contrataciones públicas, propiedad intelectual, medioambiente, asuntos laborales y solución de diferencias (capítulos 15, 18, 20, 19 y 28, respectivamente). Adicionalmente, las reglas para los servicios de telecomunicaciones, servicios financieros y comercio electrónico están contenidas en capítulos específicos (13, 11 y 14, respectivamente).

En general, estos capítulos replican los capítulos homólogos de otros acuerdos comerciales. En materia de acceso a mercados, procura eliminar o reducir sustancialmente las barreras de acceso, tanto para productos industriales como de origen agropecuario; esto incluye los productos textiles, a los cuales se les dedica un capítulo (4). En reglas de origen contiene disposiciones simples, pero que facilitan la 
acumulación de valor entre los países socios. Sobre la facilitación de comercio acordaron reglas para agilizar los procedimientos aduaneros y mejorar la transparencia y la integridad de la administración aduanera. En materia de defensa comercial, el capítulo en cuestión norma las medidas correctivas y promueve la transparencia y el debido proceso; además, incluye un mecanismo de salvaguardia de transición.

El capítulo de medidas sanitarias y fitosanitarias innova en cuanto a que permite la participación del público. El capítulo relacionado con los obstáculos técnicos pone énfasis en asegurarse de que las normas no generen barreras innecesarias al comercio. El capítulo de inversiones enfatiza en la protección básica de la inversión como el trato de nación más favorecida, y la protección contra la expropiación que no sea para fines públicos y sin el debido proceso o compensación.

El capítulo sobre contrataciones públicas busca asegurar la no discriminación y el trato nacional para proveedores de otros países del acuerdo, incluyendo especificaciones técnicas justas y objetivas. El capítulo de propiedad intelectual tiene como objetivo facilitar que las empresas logren identificar, registrar y proteger los derechos de propiedad intelectual en nuevos mercados y considera una gran variedad de instrumentos, incluyendo patentes, marcas, derechos de autor, diseños industriales, indicaciones geográficas y secretos comerciales.

Por último, los compromisos fundamentales de los capítulos sobre medioambiente y asuntos laborales son similares a los de otros acuerdos; es decir, los países se obligan a no debilitar sus normativas vigentes para lograr fines comerciales o de inversión. Sin embargo, se adicionan compromisos más amplios en ambos aspectos, como la promoción de la pesquería y la gestión forestal sostenibles y la erradicación del trabajo forzoso.

Los temas novedosos y que también tienen capítulos específicos son: la competencia (capítulo 16), el tratamiento de las empresas del 
Estado y los monopolios designados (capítulo 17), la cooperación (21), la competitividad (22), el desarrollo (23), las pequeñas y medianas empresas (24), la coherencia regulatoria (25) y la transparencia y anticorrupción (26).

\section{Las exportaciones de los países del TTP hacia Estados Unidos}

Como se discutió en la introducción, la preocupación que motiva este trabajo es dar cuenta sobre el impacto potencial que tendría el TTP sobre las exportaciones de la República Dominicana a Estados Unidos. Para ello, es útil conocer la actual estructura de las exportaciones de los países socios hacia ese mercado y la importancia relativa de cada uno.

Las exportaciones de los países del TTP se caracterizan por cuatro elementos principales, a saber:

a) Están muy concentradas en dos países: México y Canadá. En 2014, ambos explicaron el $73 \%$ de todas las exportaciones. Japón es el tercer origen en importancia de las importaciones estadounidenses desde los países del TTP, con un 15\% del total. Estos tres países, por lo tanto, explican el $88 \%$ de todas las exportaciones de los países miembros hacia Estados Unidos. Ninguno de los socios restantes rebasa el 3.5\% de las exportaciones totales.

b) Para México y Canadá el comercio con Estados Unidos tiene un elevadísimo peso. En promedio, el peso de las exportaciones hacia Estados Unidos, en las exportaciones totales de los países del TTP, alcanzó en 2014 un 31.9\%. Sin embargo, esto es explicado por el gran peso que representan esos dos países. En el caso de México, al mercado estadounidense se dirigió el $79 \%$ de sus exportaciones en 2014, mientras que Canadá envió poco menos del 76\%. En contraste, el país que le sigue en orden de importancia es Vietnam, el cual apenas colocó menos del 19\% de sus ventas en Estados 
Unidos. Japón registró un porcentaje similar, pero menos de 16\% y Chile $12.5 \%$. El resto colocó menos del $10 \%$ en ese mercado.

c) Para Estados Unidos, en 2014, las importaciones desde los países del TTP explicaron el 39\% del total; el $61 \%$ fue originario de otros países. El $40 \%$ de ese $39 \%$, un $15.5 \%$ del total, se originó en Canadá y un 35\% (13.6\% del total) en México. En conjunto, las importaciones desde México y Canadá explicaron el $75 \%$ de las importaciones estadounidenses totales desde los países del TTP, y $29 \%$ de las totales. Esto indica que, aunque los miembros del TTP son proveedores relevantes, en particular México y Canadá, Estados Unidos mantiene un importante nivel de diversificación en el origen de sus importaciones. Esto contrasta con el extraordinariamente elevado peso que para México y Canadá representa el mercado estadounidense.

d) El 79\% de las importaciones estadounidenses desde los países del TTP se realiza bajo las condiciones estipuladas en acuerdos de libre comercio, lo que representa el $31 \%$ de sus importaciones totales. Esto se deriva del hecho de que Estados Unidos ya tiene acuerdos de libre comercio con seis de los 11 países socios del TTP: México, Canadá, Singapur, Chile, Australia y Perú. No tiene acuerdos de libre comercio con Estados Unidos: Japón, Vietnam, Malasia, Nueva Zelandia y Brunei, países que explicaron el 21\% de las exportaciones de los socios del TTP a ese mercado, y el $7.9 \%$ de las importaciones totales.

En síntesis, las importaciones de Estados Unidos desde sus socios del TTP están muy concentradas en México y Canadá, países para los que el mercado estadounidense tiene un peso abrumador; en contraste, para Estados Unidos la importancia de estos es moderada. Al mismo tiempo, un muy elevado porcentaje de las importaciones de Estados Unidos desde sus socios del TTP ya se transan bajo condiciones de libre comercio, lo que supone que, en esos términos, el impacto del TTP podrían sería moderado. 


\section{Aproximación metodológica}

Los fundamentos de la metodología utilizada son relativamente estándar en este tipo de estudio y descansa en:

- la identificación de mercancías con un peso relativamente alto en las exportaciones hacia el mercado de interés (Estados Unidos);

- la identificación de países competidores en esas mercancías en Estados Unidos;

- la identificación de su nivel de competitividad en ese mercado y en el mundo;

- la cuantificación de las barreras arancelarias presentes y, por lo tanto, el mejoramiento potencial del acceso al mercado derivado de la reducción o eliminación de esas barreras;

- la medición, mediante el uso de elasticidades precio de la demanda, de la reacción de la demanda en el mercado estadounidense a la reducción de los precios como resultado de la apertura y, por lo tanto, de la desviación de la demanda desde países fuera del TTP hacia países del TTP beneficiarios de la desgravación.

El proceso se puede dividir en tres etapas:

1) Identificación de grupos agregados de productos exportados a Estados Unidos que podrían enfrentar una mayor competencia por parte de los países del TTP que no tienen acuerdos de libre comercio con Estados Unidos;

2) Identificación de grupos específicos de productos en las condiciones descritas en el punto anterior y que, a la vez, enfrentan en la actualidad aranceles positivos en el mercado estadouni- 
dense, por lo que el TTP eliminaría la desventaja relativa en materia de acceso;

3) Estimación de la reducción de precios que resultaría de la eliminación de los aranceles vigentes a favor de los socios del TTP que no tienen acuerdos de libre comercio, así como del desvío de la demanda de esos productos en Estados Unidos en beneficio de estos y en desmedro de la demanda del resto de los proveedores.

\subsection{Identificación de grupos agregados de productos bajo ame- naza potencial}

Para distinguir aquellos grupos agregados bajo potencial amenaza, se identificaron los capítulos (dos dígitos del Sistema Armonizado) de mayor importancia en las exportaciones de la República Dominicana a Estados Unidos para el período 2012-2014. Específicamente, se identificaron aquellos que registraron un valor exportado medio anual de USD 20 millones o más y que su participación en las exportaciones totales hacia ese mercado fuera mayor a $0.5 \%$ del total.

El paso inmediatamente posterior fue identificar, entre estos capítulos, aquellos en los que los países miembros del TTP registraron exportaciones a Estados Unidos equivalentes a $0.5 \%$ o más del total de sus exportaciones hacia ese mercado.

El tercer paso fue estimar el Índice de Ventajas Comparativas Reveladas (VCR) de la República Dominicana y de los países del TTP en esos capítulos. La estimación para estos últimos se realizó tanto frente al mercado mundial como frente al mercado de Estados Unidos. El VCR, desarrollado por Bela Balassa (1965), mide la especialización relativa de un país respecto al mundo en alguna mercancía o algún grupo de mercancías.

$$
\operatorname{VCR}=\left(\mathrm{X}_{\mathrm{ij}} / \mathrm{X}_{\mathrm{i}}\right) /\left(\mathrm{X}_{\mathrm{im}} / \mathrm{X}_{\mathrm{m}}\right)
$$


donde $\mathrm{X}_{\mathrm{ij}}$ es el valor exportado de la mercancía $\mathrm{j}$ por el país $\mathrm{i}$; $\mathrm{X}_{\mathrm{i}}$ es el valor de las exportaciones totales del país $\mathrm{i} ; \mathrm{X}_{\mathrm{im}}$ es el valor de las exportaciones mundiales de la mercancía $\mathrm{j}$; $\mathrm{y}_{\mathrm{m}}$ es el valor de las exportaciones mundiales de todas las mercancías. Si VCR $>1$ esto sugeriría que el país i está relativamente especializado en la mercancía $\mathrm{j}$, porque el peso de las exportaciones de esa mercancía en las exportaciones totales del país es superior a la media mundial. Por el contrario, si VCR $<1$ el grado de especialización de ese país en esa mercancía o grupo de mercancías es menor que el promedio mundial.

En un contexto de libre comercio, si el país es relativamente más especializado que el resto del mundo en esa mercancía, se presume que el país tiene ventajas comparativas en ella, porque el comercio así lo "revela". Es precisamente en este supuesto que reside la limitación del VCR como indicador de ventajas comparativas. Mientras que existan barreras para el comercio y en la medida en que los patrones de comercio no respondan a las ventajas comparativas sino a otros factores como la presencia (o ausencia) de economías de escala o la presencia de barreras de entrada en actividades específicas, el indicador se hace menos efectivo para lograr una aproximación al nivel de competitividad del país en ese producto.

Es bien conocido que el comercio internacional está muy influenciado por barreras arancelarias y de otro tipo, y que además de las ventajas comparativas y la dotación de recursos, las economías de escala juegan un rol importante en la conformación de los patrones de comercio; sin embargo, hay que reconocer que, a lo largo de las últimas décadas, las barreras impuestas al comercio por los Estados se han venido reduciendo de forma decidida. En la medida en que las barreras se reducen, el indicador podría reflejar mejor las ventajas comparativas de un país en un producto determinado. Adicionalmente, independientemente de cuál sea la fuente de especialización, las ventajas comparativas o la presencia de economías de escala, un VCR mayor que uno supondría una ventaja relativa de ese país en ese producto. 
Finalmente, se identificaron los países que ya tenían un tratado de libre comercio con Estados Unidos y los productos exportados a ese mercado de países sin tratado, pero sujetos a un arancel mayor que $0 \%$. Estos productos y países fueron excluidos a fin de reconocer aquellos grupos de productos que cumplen con tres condiciones simultáneas:

- Son relevantes en la canasta de exportación hacia Estados Unidos, tanto del país como de los potenciales competidores.

- Son competitivos de acuerdo al ICV, tanto para la República Dominicana como para los países del TTP.

- Verían mejorar sus condiciones de acceso al mercado de Estados Unidos como resultado de la entrada en vigor del TTP; específicamente, verían reducir a $0 \%$ el arancel.

\subsection{Identificación de grupos específicos de productos bajo ame- naza potencial}

Un segundo análisis se desarrolló a nivel de partidas (4 dígitos) del Sistema Armonizado. La motivación del análisis a ese nivel de desagregación es que los capítulos tienen un alto nivel de agregación, por lo que hay una alta heterogeneidad en las mercancías y en los procesos productivos. Esto hace que los promedios escondan una variedad de situaciones. El análisis a ese nivel es relevante para una primera aproximación, pero se requiere uno a un nivel mayor de desagregación que identifique los productos específicos que son relevantes en la canasta exportadora dominicana, y entre estos, aquellos en los que tanto la República Dominicana como los países del TTP "revelan" ventajas.

A este nivel, se llevó a cabo un procedimiento similar al seguido en análisis a nivel de capítulos (dos dígitos). Se seleccionaron las partidas de mayor peso en las exportaciones de la República Dominicana 
a Estados Unidos, específicamente, aquellas que registraran un valor medio anual para el período 2012-2014 superior a USD 10 millones.

El segundo paso fue estimar los IVCR para cada una de esas partidas, tanto para la República Dominicana como para los países del TTP competidores en el mercado de Estados Unidos. De estos se seleccionaron aquellos en los que los países competidores tuvieran un ICVR superior a 1.0. respecto al mundo o al mercado de Estados Unidos.

El tercero fue eliminar de la lista los productos de países que ya tienen un tratado de libre comercio con Estados Unidos, asumiendo que sus exportaciones no enfrentan barreras arancelarias en ese mercado o están en proceso de ser eliminadas.

El cuarto y último paso fue eliminar de la lista los productos de los países que, aun no teniendo tratado de libre comercio con Estados Unidos, están sujetos a una tasa arancelaria de $0 \%$ en ese mercado.

\subsection{Estimación del desvío de demanda como resultado de la desgravación arancelaria}

Se asumió que el TTP considera, para cada una de las partidas seleccionadas originarias de todos los países socios, un desmonte arancelario total en el mercado de Estados Unidos y que esa reducción se traduciría en una disminución de igual magnitud en el precio de venta.

Adicionalmente, se utilizaron estimaciones de la elasticidad precio de la demanda en Estados Unidos para mercancías codificadas a 6 dígitos del Sistema Armonizado (subpartidas) para reestimar elasticidades agregadas a 4 dígitos. Para ello, se usó el promedio simple.

Finalmente, utilizando las reducciones arancelarias y las elasticidades de demanda, se estimó la desviación de la demanda en Estados Unidos a favor de productos originarios de los países del TTP beneficiarios del desmonte arancelario. El objetivo es dimensionar 
la reducción potencial de la demanda que se registrarían en esos productos que son originarios de países no beneficiarios del TTP.

\section{Identificando la competencia de la República Dominicana en el mercado de Estados Unidos}

Este apartado tiene el propósito de presentar los resultados del proceso seguido para identificar los grupos de mercancías exportadas a Estados Unidos desde la República Dominicana que podrían ser negativamente afectados en caso de que el TTP entre en efecto.

Para empezar, hay que indicar que a pesar de que el peso del mercado estadounidense en las exportaciones totales de bienes del país ha declinado en los últimos años, este continúa siendo el más importante de los mercados externos. Entre 2012 y 2014, las exportaciones hacia ese mercado explicaron el $51.4 \%$ de las exportaciones totales de bienes en ese período. Por tal razón, lo que pase en ese mercado, incluyendo nuevos acuerdos comerciales, es de mucha relevancia para el sector externo dominicano.

Por otra parte, en el cuadro 1 se identifica los 21 capítulos del Sistema Armonizado de mayor peso en las exportaciones dominicanas de bienes hacia Estados Unidos, con un valor de US\$ 20 millones o más o $0.5 \%$ de las exportaciones totales hacia ese mercado. En conjunto, estas representaron el $94 \%$ de las exportaciones totales hacia Estados Unidos. 
Cuadro 1. Principales exportaciones de bienes de la República Dominicana hacia Estados Unidos. Miles de US\$, Promedio anual 2012-2014.

\begin{tabular}{|c|c|c|c|}
\hline \multirow{2}{*}{$\begin{array}{c}\text { Capítulo } \\
\text { SA }\end{array}$} & \multirow{2}{*}{ Descripción } & \multicolumn{2}{|c|}{$\begin{array}{l}\text { Exportaciones a } \\
\text { EEUU }\end{array}$} \\
\hline & & $\begin{array}{c}\text { Valor } \\
\text { (miles US\$) }\end{array}$ & $\%$ \\
\hline 90 & $\begin{array}{l}\text { Instrumentos, aparatos de óptica, fotografía, } \\
\text { cinematografía, medida, control }\end{array}$ & 722,377 & $16.8 \%$ \\
\hline 85 & $\begin{array}{l}\text { Máquinas, aparatos y material eléctrico, sus } \\
\text { partes; aparatos de grabación }\end{array}$ & 518,902 & $12.1 \%$ \\
\hline 71 & $\begin{array}{l}\text { Perlas finas o cultivadas, piedras preciosas, } \\
\text { semipreciosas y similares }\end{array}$ & 493,169 & $11.5 \%$ \\
\hline 24 & Tabaco y sucedáneos del tabaco elaborados & 469,597 & $10.9 \%$ \\
\hline 61 & Prendas y complementos de vestir, de punto & 313,709 & $7.3 \%$ \\
\hline 64 & $\begin{array}{l}\text { Calzado, polainas, botines y artículos aná- } \\
\text { logos y sus partes }\end{array}$ & 281,214 & $6.6 \%$ \\
\hline 62 & $\begin{array}{l}\text { Prendas y complementos de vestir, excepto } \\
\text { los de punto }\end{array}$ & 279,450 & $6.5 \%$ \\
\hline 30 & Productos farmacéuticos & 191,061 & $4.5 \%$ \\
\hline 39 & $\begin{array}{l}\text { Materias plásticas y manufacturas de estas } \\
\text { materias }\end{array}$ & 149,480 & $3.5 \%$ \\
\hline 17 & Azúcares y artículos de confitería & 84,308 & $2.0 \%$ \\
\hline 84 & $\begin{array}{l}\text { Máquinas, reactores nucleares, calderas, } \\
\text { aparatos y artefactos mecánicos. }\end{array}$ & 76,634 & $1.8 \%$ \\
\hline 63 & $\begin{array}{l}\text { Los demás artículos textiles confecciona- } \\
\text { dos; conjuntos/surtidos... }\end{array}$ & 74,247 & $1.7 \%$ \\
\hline 18 & Cacao y sus preparaciones & 72,909 & $1.7 \%$ \\
\hline 07 & $\begin{array}{l}\text { Legumbres y hortalizas, plantas, raíces y } \\
\text { tubérculos alimenticios }\end{array}$ & 53,538 & $1.2 \%$ \\
\hline 33 & $\begin{array}{l}\text { Aceites esenciales y resinoides; prep. de } \\
\text { perfumería, de tocador }\end{array}$ & 50,318 & $1.2 \%$ \\
\hline 72 & Fundición, hierro y acero & 45,324 & $1.1 \%$ \\
\hline 21 & Preparaciones alimenticias diversas & 40,238 & $0.9 \%$ \\
\hline 22 & Bebidas, líquidos alcohólicos y vinagre & 35,751 & $0.8 \%$ \\
\hline 08 & $\begin{array}{l}\text { Frutos comestibles; cortezas de agrios o de } \\
\text { melones }\end{array}$ & 34,995 & $0.8 \%$ \\
\hline 49 & $\begin{array}{l}\text { Productos editoriales, de la prensa/de otras } \\
\text { industrias gráficas }\end{array}$ & 20,904 & $0.5 \%$ \\
\hline 83 & Manufacturas diversas de metales comunes & 20,808 & $0.5 \%$ \\
\hline
\end{tabular}

Fuente: elaborado con información de UNCOMTRADE. 
Se aprecia que instrumentos y aparatos médicos (del capítulo 30), aparatos eléctricos (del 85), oro (del 71), cigarros y puros (del 24), confecciones de vestir de punto (del 61), calzados y partes (del 61), prendas y confecciones no de punto (del 62) y productos farmacéuticos (del 30) explicaron el 76.2\% de las exportaciones totales a ese mercado. Son mercancías producidas casi totalmente en parques de zonas francas de exportación.

\subsection{Presencia en el mercado de EEUU y ventaja comparativa revelada}

En este punto, la pregunta obligada es cuáles de esas exportaciones podrían ver intensificada la competencia como resultado del TTP. Una primera aproximación busca identificar en cuáles países del TTP las exportaciones de esos productos hacia EEUU tienen un peso relevante y cuáles de esos países muestran tener ventajas comparativas.

En el cuadro 2 se toman los capítulos listados en el cuadro 1 y se indica, para cada uno de ellos: a) el número de países del TTP (para un total de 11 países, esto es, todos excluyendo EEUU) en los cuales las exportaciones de mercancías de esos capítulos explican el $0.5 \%$ o más de sus exportaciones totales a ese mercado (tercera columna); b) el número de países del TTP en los cuales el valor de las exportaciones hacia EEUU para el período considerado supera el valor de las exportaciones dominicanas hacia ese mercado; y c) el número de países del TTP que tiene VCR $>1$ en el mercado de EEUU o en el mundo.

Como se advierte, un número relativamente elevado de países del TTP parece tener presencia relevante en el mercado de EEUU o ventaja comparativa en los capítulos relevantes para la República Dominicana. En cada uno de esos capítulos, un promedio de cinco de los 11 países del TTP tiene exportaciones al mercado de EEUU que representan $0.5 \%$ o más de sus exportaciones totales a ese mercado. Además, en cada capítulo, un promedio de 4.9 países (de 11) registra un valor de las exportaciones superior al valor de las exportaciones 
dominicanas. Por último, en cada capítulo, un promedio de 3.6 países muestra tener ventaja comparativa.

Cuadro 2. Principales exportaciones dominicanas a EEUU y potenciales países COMPETIDORES MIEMBROS DEL TTP EN ESE MERCADO (DATOS BASADOS EN EL PERÍODO 2012-2014)

\begin{tabular}{|c|c|c|c|c|}
\hline $\begin{array}{l}\text { SA (dos } \\
\text { dígitos) }\end{array}$ & Descripción & $\begin{array}{c}\text { Importancia de } \\
\text { las exportacio- } \\
\text { nes de países } \\
\text { TTP en mercado } \\
\text { de EEUU } \\
(>0.5 \% \text { del total })\end{array}$ & $\begin{array}{c}\text { Total de } \\
\text { expor- } \\
\text { taciones } \\
\text { comparado } \\
\text { con Rep. } \\
\text { Dominica- } \\
\text { na }(>1)\end{array}$ & $\begin{array}{r}\text { Con } \\
\text { VCR }\end{array}$ \\
\hline & & \multicolumn{3}{|c|}{ \# de países: 11} \\
\hline 90 & $\begin{array}{l}\text { Instrumentos de óptica o pre- } \\
\text { cisión }\end{array}$ & 9 & 6 & 7 \\
\hline 85 & $\begin{array}{l}\text { Máquinas, aparatos y material } \\
\text { eléctrico }\end{array}$ & 10 & 6 & 4 \\
\hline 71 & $\begin{array}{l}\text { Perlas finas o cultivadas, pie- } \\
\text { dras preciosas }\end{array}$ & 7 & 4 & 3 \\
\hline 24 & $\begin{array}{l}\text { Tabaco y sucedáneos del tabaco } \\
\text { elaborados }\end{array}$ & & 9 & \\
\hline 61 & $\begin{array}{l}\text { Prendas y complementos de } \\
\text { vestir, de punto }\end{array}$ & 6 & 5 & 3 \\
\hline 64 & $\begin{array}{l}\text { Calzado, polainas, botines y } \\
\text { análogos }\end{array}$ & 1 & 2 & 2 \\
\hline 62 & $\begin{array}{l}\text { Prendas de vestir, excepto los } \\
\text { de punto }\end{array}$ & 4 & 3 & 1 \\
\hline 30 & Productos farmacéuticos & 5 & 5 & 1 \\
\hline 39 & $\begin{array}{l}\text { Materias plásticas y manufac- } \\
\text { turas }\end{array}$ & 8 & 6 & 4 \\
\hline 17 & $\begin{array}{l}\text { Azúcares y artículos de confi- } \\
\text { tería }\end{array}$ & & 2 & 5 \\
\hline 84 & $\begin{array}{l}\text { Máquinas, aparatos y artefactos } \\
\text { mecánicos }\end{array}$ & 11 & 9 & 3 \\
\hline 63 & Los demás artículos textiles & & 3 & 1 \\
\hline 18 & Cacao y preparaciones de cacao & 1 & 3 & 4 \\
\hline 7 & $\begin{array}{l}\text { Legumbres y hortalizas, plan- } \\
\text { tas, raíces }\end{array}$ & 2 & 3 & 6 \\
\hline 33 & Aceites esenciales; perfumes & 3 & 4 & 3 \\
\hline 72 & Fundición, hierro y acero & 6 & 6 & 2 \\
\hline
\end{tabular}




\begin{tabular}{|c|c|c|c|c|}
\hline 21 & $\begin{array}{l}\text { Preparaciones alimenticias } \\
\text { diversas }\end{array}$ & & 3 & 7 \\
\hline 22 & $\begin{array}{l}\text { Bebidas, líquidos alcohólicos y } \\
\text { vinagre }\end{array}$ & 4 & 6 & 5 \\
\hline 08 & Frutos comestibles & 6 & 7 & 6 \\
\hline 49 & $\begin{array}{l}\text { Productos editoriales. De indus- } \\
\text { trias gráficas }\end{array}$ & 1 & 5 & 3 \\
\hline 83 & $\begin{array}{l}\text { Manufacturas diversas de meta- } \\
\text { les comunes }\end{array}$ & 1 & 6 & 1 \\
\hline & Promedio & 5.0 & 4.9 & 3.6 \\
\hline
\end{tabular}

Fuente: elaborado con información de UNCOMTRADE.

Para el capítulo 90, por ejemplo, el de mayor peso en las exportaciones dominicanas a EEUU, 9 países del TTP (de un total de 11) registran exportaciones hacia ese país y estas representaron el $0.5 \%$ o más de las exportaciones hacia ese mercado; en el capítulo 85, ese fue el caso para 10 de 11 países; y en el capítulo 71, 6 de 11 países.

Cuando el ejercicio se hace para países del TTP que no tienen acuerdos de libre comercio con EEUU y, por lo tanto, verían mejorado su acceso a ese mercado como resultado del acuerdo, los resultados son similares. En los únicos capítulos en los que no hay presencia de países del TTP sin acuerdos de libre comercio y que no tienen ventaja comparativa en los capítulos relevantes para República Dominicana son en el capítulo 24 (tabaco y sucedáneos) y en el capítulo 33 (aceites esenciales; perfumes). Por ejemplo, en los dos primeros capítulos todos los países del TTP sin acuerdos de libre comercio con EEUU tienen presencia relevante en ese mercado; tres de los cinco registraron exportaciones por un valor superior al de República Dominicana; y en el primer capítulo (90), cuatro de cinco muestran tener ventaja comparativa. 
Cuadro 3. Principales exportaciones dominicanas a EEUU y potenciales países COMPETIDORES MIEMBROS DEL TTP EN ESE MERCADO QUE NO TIENEN ACUERDOS DE LIBRE comercio con EEUU. Datos basados en el PERÍodo 2012-2014.

\begin{tabular}{|c|c|c|c|c|}
\hline $\begin{array}{c}\text { SA } \\
\text { (dos } \\
\text { dígitos) }\end{array}$ & Descripción & $\begin{array}{l}\text { Importancia de las } \\
\text { exportaciones de } \\
\text { países TTP en mer- } \\
\text { cado de EEUU } \\
(>0.5 \% \text { del total })\end{array}$ & $\begin{array}{c}\text { Total exportacio- } \\
\text { nes comparado con } \\
\text { Rep. Dominicana } \\
(>1)\end{array}$ & $\begin{array}{l}\text { Con } \\
\text { VCR }\end{array}$ \\
\hline & & \multicolumn{3}{|c|}{ \# de países: 5} \\
\hline 90 & $\begin{array}{l}\text { Instrumentos de óptica } \\
\text { o precisión }\end{array}$ & 5 & 3 & 4 \\
\hline 85 & $\begin{array}{l}\text { Máquinas, aparatos y } \\
\text { material eléctrico }\end{array}$ & 5 & 3 & 2 \\
\hline 71 & $\begin{array}{l}\text { Perlas finas o cultiva- } \\
\text { das, piedras preciosas }\end{array}$ & 1 & 1 & \\
\hline 24 & $\begin{array}{l}\text { Tabaco y sucedáneos } \\
\text { del tabaco elaborados }\end{array}$ & & & \\
\hline 61 & $\begin{array}{l}\text { Prendas y complemen- } \\
\text { tos de vestir, de punto }\end{array}$ & 3 & 2 & 2 \\
\hline 64 & $\begin{array}{l}\text { Calzado, polainas, boti- } \\
\text { nes y análogos }\end{array}$ & 1 & 1 & 1 \\
\hline 62 & $\begin{array}{l}\text { Prendas de vestir, ex- } \\
\text { cepto los de punto }\end{array}$ & 3 & 1 & 1 \\
\hline 30 & $\begin{array}{l}\text { Productos farmacéu- } \\
\text { ticos }\end{array}$ & 2 & 1 & \\
\hline 39 & $\begin{array}{l}\text { Materias plásticas y } \\
\text { manufacturas }\end{array}$ & 3 & 3 & 2 \\
\hline 17 & $\begin{array}{l}\text { Azúcares y artículos de } \\
\text { confitería }\end{array}$ & & & 1 \\
\hline 84 & $\begin{array}{l}\text { Máquinas, aparatos y } \\
\text { artefactos mecánicos }\end{array}$ & 5 & 3 & 2 \\
\hline 63 & $\begin{array}{l}\text { Los demás artículos } \\
\text { textiles }\end{array}$ & & 1 & 1 \\
\hline 18 & $\begin{array}{l}\text { Cacao y preparaciones } \\
\text { de cacao }\end{array}$ & 1 & 1 & 2 \\
\hline 7 & $\begin{array}{l}\text { Legumbres y hortalizas, } \\
\text { plantas, raíces }\end{array}$ & & & 2 \\
\hline 33 & $\begin{array}{l}\text { Aceites esenciales; } \\
\text { perfumes }\end{array}$ & & & \\
\hline 72 & $\begin{array}{l}\text { Fundición, hierro y } \\
\text { acero }\end{array}$ & 2 & 2 & 1 \\
\hline 21 & $\begin{array}{l}\text { Preparaciones alimenti- } \\
\text { cias diversas }\end{array}$ & & 1 & 2 \\
\hline
\end{tabular}




$\begin{array}{rlccc} & \text { Bebidas, líquidos alco- } & & & \\ 22 & \begin{array}{l}\text { hólicos y vinagre } \\ 08\end{array} & 1 & 2 & 2 \\ & \begin{array}{l}\text { Frutos comestibles } \\ \text { Productos editoriales. }\end{array} & 2 & 2 & \\ 49 & \begin{array}{l}\text { De industrias gráficas } \\ \text { Manufacturas diversas } \\ 83\end{array} & & 2 & \\ \text { de metales comunes } & \text { Promedio } & 2.6 & 1.8 & 1.8\end{array}$

Fuente: elaborado con información de UNCOMTRADE.

Como se discutió en la sección 4.2., en la medida en que a nivel de capítulos existe una alta heterogeneidad en las mercancías y en los procesos productivos, se requiere de un análisis a un menor nivel de agregación que permita identificar, entre los capítulos en los que los países del TTP registran presencia en el mercado de EEUU, aquellos productos o grupos de productos específicos relevantes en la canasta exportadora dominicana en los que los países del TTP muestren ventajas comparativas reveladas.

El cuadro 4 lista las 37 partidas de mayor peso en las exportaciones dominicanas a EEUU, cuyo valor registrado de exportaciones medias anuales entre 2012 y 2014 fue superior a US\$ 10 millones. La tercera columna presenta el valor de las exportaciones; la cuarta muestra la participación en las exportaciones hacia EEUU; y la quinta, el Índice de Ventajas Comparativas Reveladas (IVCR).

Entre 2012 y 2014, el valor medio anual de estas exportaciones ascendió a US\$ 3,172.3 millones, lo que fue equivalente al 74\% de todas las exportaciones a ese mercado.

De esos 37 capítulos, solo en uno (6110, Suéteres "jerseys", "pullovers", cardiganes, chalecos y artículos de punto) el país no registró un IVCR mayor a 1. En el resto, la evidencia indica que el país tiene ventaja comparativa en esos productos. En 16 capítulos el IVCR es de dos dígitos, lo que sugiere una elevada especialización en ellos, comparado con el resto del mundo. Entre estos se destacan productos de 
tabaco (2402 y 2403), confecciones de diversos tipos (6109 y 6212), azúcar y melaza (1701 y 1703), cacao (1801), calzados y partes (6405 y 6406), artículos de joyería y sus partes (7113), y preparaciones y artículos farmacéuticos (3006).

Cuadro 4. Principales exportaciones de República Dominicana a EeUU, por partida del Sistema ARmonizado (Descripción, VAlor EN Miles de US\$ y participación). Período 2012-2014.

\begin{tabular}{|c|c|c|c|c|}
\hline \multirow{3}{*}{ Partida } & \multirow{3}{*}{ Descripción de la partida } & \multicolumn{2}{|c|}{$\begin{array}{l}\text { Exportaciones a } \\
\text { EEUU }\end{array}$} & \multirow[t]{3}{*}{ IVCR } \\
\hline & & \multirow[b]{2}{*}{$\begin{array}{l}\text { Miles de } \\
\text { US\$ }\end{array}$} & \multirow{2}{*}{$\begin{array}{l}\% \text { del } \\
\text { total } \\
\text { a } \\
\text { EEUU }\end{array}$} & \\
\hline & & & & \\
\hline 9018 & $\begin{array}{l}\text { Instrumentos y aparatos de medicina, cirugía, odontolo- } \\
\text { gía o veterinaria }\end{array}$ & 679,222 & $15.8 \%$ & 20.2 \\
\hline 2402 & $\begin{array}{l}\text { Cigarros "puros", incl. despuntados, cigarritos "puritos" } \\
\text { y cigarrillos }\end{array}$ & 430,596 & $10.0 \%$ & 225.5 \\
\hline 7108 & $\begin{array}{l}\text { Oro, incl. el oro platinado, en bruto, semilabrado o en } \\
\text { polvo }\end{array}$ & 272,799 & $6.4 \%$ & 9.9 \\
\hline 6109 & T-shirts y camisetas, de punto & 223,976 & $5.2 \%$ & 22.4 \\
\hline 7113 & $\begin{array}{l}\text { Artículos de joyería y sus partes, de metal precioso o } \\
\text { chapado }\end{array}$ & 191,804 & $4.5 \%$ & 14.0 \\
\hline 3006 & Preparaciones y artículos farmacéuticos & 187,892 & $4.4 \%$ & 44.3 \\
\hline 6403 & $\begin{array}{l}\text { Calzado con suela de caucho o plástico y parte superior } \\
\text { de materia distinto del caucho, el plástico, el cuero natu- } \\
\text { ral o las materias textiles }\end{array}$ & 146,855 & $3.4 \%$ & 6.4 \\
\hline 6405 & $\begin{array}{l}\text { Calzado con suela de caucho o plástico y parte superior } \\
\text { de materia distinto del caucho, el plástico, el cuero natu- } \\
\text { ral o las materias textiles }\end{array}$ & 110,112 & $2.6 \%$ & 85.3 \\
\hline 6203 & $\begin{array}{l}\text { Trajes "ambos o ternos", conjuntos, chaquetas "sacos", } \\
\text { pantalones largos, pantalones con peto, pantalones cortos } \\
\text { "calzones" y "shorts" }\end{array}$ & 107,861 & $2.5 \%$ & 6.7 \\
\hline 6212 & $\begin{array}{l}\text { Sostenes "corpiños", fajas, corsés, tirantes "tiradores", } \\
\text { ligas y artículos simil. y sus partes, de todo tipo de mate- } \\
\text { ria textil, incl. elásticas y de punto }\end{array}$ & 100,640 & $2.3 \%$ & 21.6 \\
\hline 1701 & $\begin{array}{l}\text { Azúcar de caña o de remolacha y sacarosa químicamente } \\
\text { pura, en estado sólido }\end{array}$ & 71,941 & $1.7 \%$ & 21.1 \\
\hline 1801 & Cacao en grano, entero o partido, crudo o tostado & 66,246 & $1.5 \%$ & 31.1 \\
\hline 6307 & $\begin{array}{l}\text { Artículos de materia textil, confeccionados, incl. los } \\
\text { patrones para prendas de vestir, n.c.o.p. }\end{array}$ & 62,869 & $1.5 \%$ & 9.2 \\
\hline 3921 & Polímeros de etileno, en formas primarias & 38,252 & $0.9 \%$ & 10.5 \\
\hline 3926 & $\begin{array}{l}\text { Manufacturas de plástico y manufacturas de las demás } \\
\text { materias de las partidas } 3901 \text { a 3914, n.c.o.p. }\end{array}$ & 36,777 & $0.9 \%$ & 2.4 \\
\hline
\end{tabular}


Artículos para transporte o envasado, de plástico; tapones, tapas, cápsulas y demás dispositivos de cierre, de

Polarímetros, refractómetros, espectrómetros, analizadores de gases o de humos

$32,087 \quad 0.7 \% \quad 3.2$

Camisas para hombres o niños (exc. de punto, camisones 6205 y camisetas)

$27,600 \quad 0.6 \% \quad 4.1$

6108 Combinaciones, enaguas, bragas "bombachas, calzones"

Preparaciones para higiene bucal o dental, incl. los pol-

3306 vos y cremas para la adherencia de las dentaduras

$26,620 \quad 0.6 \% \quad 37.1$

0709 patatas "papas", tomates, hortalizas aliáceas, coles

$25,263 \quad 0.6 \% \quad 5.5$

2106 Preparaciones alimenticias, n.c.o.p.

$25,123 \quad 0.6 \% \quad 6.9$

2401 Tabaco en rama o sin elaborar; desperdicios de tabaco

Desperdicios y desechos, de metal precioso o chapado de

Vajilla y demás artículos de uso doméstico y artículos de

Dátiles, higos, piñas "ananás", aguacates "paltas", gua-

0804 yabas, mangos y mangostanes, frescos o secos

$18,637 \quad 0.4 \% \quad 4.5$

Suéteres "jerseys", "pullovers", cardiganes, chalecos y

6110 artículos de punto

$\begin{array}{lll}18,341 \quad 0.4 \% & 0.7\end{array}$

Sellos "estampillas" de correos, timbres fiscales y análogos, sin obliterar, que tengan o estén destinados a tener

Preparaciones de belleza, maquillaje y para el cuidado

Preparaciones para salsas y salsas preparadas; condimen-

Candados, cerraduras y cerrojos "de llave, de combina-

8301 ción o eléctricos", de metal

Tabacos y sucedáneos del tabaco, elaborados; tabaco

2403 "homogeneizado" o "reconstituido"

Trajes "ambos o ternos", conjuntos, chaquetas "sacos", pantalones largos, pantalones con peto, pantalones cortos

Abrigos, chaquetones, capas, anoraks, cazadoras y artí-

6102 culos simil., de punto, para mujeres o niñas

Melaza procedente de la extracción o del refinado de

$10,271 \quad 0.2 \% \quad 35.4$

Total

$3,172,252 \quad 74.0 \% \quad \ldots$

Fuente: elaborado con información de UNCOMTRADE. 
Pero, ¿en cuáles de esos capítulos los países del TTP que no tiene acuerdos de libre comercio con Estados Unidos tienen ventaja comparativa?

El cuadro 5 responde la pregunta anterior y revela que los países del TTP sin acuerdos de libre comercio con EEUU tienen ventaja comparativa, globalmente o en el mercado de EEUU, en 21 de las 37 partidas más relevantes que la República Dominicana exporta a ese mercado, y en 23 de las 27 de mayor peso.

Cuadro 5. Índice de Ventajas Comparativas Reveladas (IVCR) de los países DEL TTP SIN ACUERDOS DE LIBRE COMERCIO CON EEUU, EN PARTIDAS ARANCELARIAS DE MAYOR PESO EN LAS EXPORTACIONES DOMINICANAS A EEUU.

\begin{tabular}{|c|c|c|c|c|c|c|c|}
\hline $\begin{array}{l}\text { Parti- } \\
\text { da }\end{array}$ & Descripción de la partida & $\begin{array}{c}\text { Bru- } \\
\text { nei }\end{array}$ & Japón & $\begin{array}{l}\text { Mala- } \\
\text { sia }\end{array}$ & $\begin{array}{c}\text { Nue- } \\
\text { va } \\
\text { Ze- } \\
\text { landia }\end{array}$ & $\begin{array}{l}\text { Sin- } \\
\text { gapur }\end{array}$ & Vietnam \\
\hline 9018 & $\begin{array}{l}\text { Instrumentos y aparatos de } \\
\text { medicina, cirugía }\end{array}$ & & & & & & \\
\hline 2402 & $\begin{array}{l}\text { Cigarros "puros", incl. des- } \\
\text { puntados, cigarritos }\end{array}$ & & & & 3.51 & & \\
\hline 7108 & $\begin{array}{l}\text { Oro, incl. el oro platinado, en } \\
\text { bruto, semilabrado }\end{array}$ & & & & 2.42 & & 1.27 \\
\hline 6109 & T-shirts y camisetas, de punto & & & & 6.33 & & 4.09 \\
\hline 7113 & $\begin{array}{l}\text { Artículos de joyería y sus } \\
\text { partes, de metal precioso }\end{array}$ & & & & & & 4.62 \\
\hline 3006 & $\begin{array}{l}\text { Preparaciones y artículos } \\
\text { farmacéuticos }\end{array}$ & & & & & & \\
\hline 6403 & $\begin{array}{l}\text { Calzado con suela de caucho } \\
\text { o plástico y parte... }\end{array}$ & & & & 1.28 & & \\
\hline 6405 & $\begin{array}{l}\text { Calzado con suela de caucho } \\
\text { o plástico y parte... }\end{array}$ & & & 4.25 & 1.06 & & \\
\hline 6203 & $\begin{array}{l}\text { Trajes "ambos o ternos", } \\
\text { conjuntos, chaquetas... }\end{array}$ & & & & 1.97 & & \\
\hline 6212 & $\begin{array}{l}\text { Sostenes "corpiños", fajas, } \\
\text { corsés, tirantes "tiradores" }\end{array}$ & & & 1.58 & 4.82 & & \\
\hline 1701 & $\begin{array}{l}\text { Azúcar de caña o de remola- } \\
\text { cha y sacarosa }\end{array}$ & & & & & & \\
\hline 1801 & $\begin{array}{l}\text { Cacao en grano, entero o } \\
\text { partido, crudo o tostado }\end{array}$ & & & & & 2.46 & \\
\hline
\end{tabular}


Artículos de materia textil,

6307 confeccionados

1.95

$1.12 \quad 1.88$

1.24

Polímeros de etileno, en for-

3921 mas primarias

1.88

3926 Manufacturas de plástico...

1.26

4.41

Artículos para transporte o

3923 envasado, de plástico...

Polarímetros, refractómetros,

9027 espectrómetros..

6.32

Camisas para hombres o

6205 niños (exc. de punto)...

Combinaciones, enaguas,

6108 bragas bombachas..

1.08

Preparaciones para higiene

3306 bucal o dental..

Hortalizas, incl. "silvestres",

0709 frescas o refrigeradas..

1.15

Preparaciones alimenticias,

2106 n.c.o.p.

Tabaco en rama o sin elabo-

2401 rar; desperdicios de tabaco

Partes de calzado, incl. las

6406 partes superiores fijadas..

Desperdicios y desechos, de

7112 metal precioso...

Vajilla y demás artículos ...

3924 de plástico

Dátiles, higos, piñas "ana-

0804 nás”, aguacates "paltas”...

Suéteres "jerseys", "pullo-

6110 vers", cardiganes, chalecos...

Sellos "estampillas" de co-

4907 rreos, timbres fiscales...

Preparaciones de belleza,

3304 maquillaje...

Preparaciones para salsas y

2103 salsas preparadas...

Candados, cerraduras y ce-

8301 rrojos..

Tabacos y sucedáneos del

2403 tabaco, elaborados...

Trajes "ambos o ternos",

6103 conjuntos, chaquetas... 


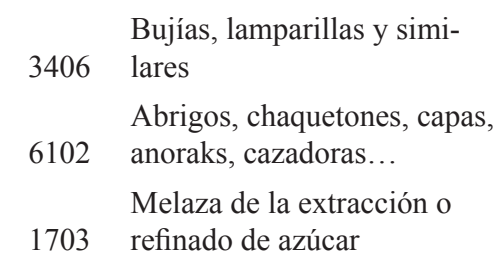

Nota. Solo se muestran los IVCR $>1$

Fuente: elaborado con información de UNCOMTRADE y OEA-SICE http://www.sice.oas. org/ctyindex/USA/USAagreements_s.asp.

El valor medio anual de esas exportaciones dominicanas, que enfrentaría una competencia intensificada en el mercado de EEUU por parte de países del TTP, suma US\$ 1.6 mil millones y representa el $37 \%$ de exportaciones totales. Las excepciones más notables son productos farmacéuticos, cacao, azúcar y otras exportaciones con montos relativamente bajos, en las que esos países no parecen tener ventajas comparativas.

Algunos de los resultados más destacados de este cuadro son los siguientes:

- En general, la potencial amenaza en cada partida provendría de más de un país;

- Vietnam y Nueva Zelandia se destacan por el número de partidas en las que tienen ventajas comparativas;

- Vietnam muestra tener ventajas comparativas en instrumentos y aparatos médicos, oro, artículos de joyería, confecciones textiles, plásticos, hortalizas, preparaciones alimenticias, partes de calzados y tabaco en rama;

- Nueva Zelandia muestra tener ventajas comparativas en instrumentos y aparatos médicos, cigarros y puros, oro, artículos de joyería, confecciones textiles, calzado y sus partes, preparaciones para la higiene bucal o dental, y algunas frutas; 
- Las exportaciones de cigarros y puros, con las que la República Dominicana tiene un fuerte dominio del mercado estadounidense y cuyo número de competidores es relativamente reducido, llevaría al país a enfrentar una potencial competencia de Nueva Zelandia.

\subsection{Presencia de barreras arancelarias}

Para que un país competidor en el mercado de EEUU incremente su participación por efectos de un acuerdo comercial a costa de otros, no solo es necesario que ese país tenga ventajas en el producto de que se trate, sino que se precisa que dicho acuerdo mejore el acceso a ese mercado; específicamente, que elimine las barreras arancelarias a dicho producto. Eso solo es posible cuando el producto, antes del acuerdo, está sujeto a aranceles mayores a $0 \%$ y que el acuerdo los elimine.

En el caso que nos ocupa, hacer esta aproximación requiere identificar, entre los productos relevantes para la República Dominicana en el mercado de EEUU y en los cuales los países del TTP tienen ventaja comparativa, aquellos para los que el arancel de Nación Más Favorecida (NMF) de EEUU es mayor que cero. El arancel NMF es una definición usada en el marco de la Organización Mundial del Comercio (OMC) y se refiere al arancel que un país miembro aplica a mercancías de países miembros de la organización pero que no tienen acuerdos comerciales.

En el cuadro 6 se selecciona las 21 partidas del cuadro 5 para los que el IVCR fue mayor que 1 en al menos algún país del TTP; y de estas se selecciona, a su vez, solo aquellas para las que el arancel NMF es mayor a $0 \%$. El resultado es un total de 13 partidas arancelarias que cumplen, simultáneamente, con tres condiciones:

a) tienen un peso relevante en las exportaciones dominicanas a EEUU; 
b) observan en los países del TTP que no tienen acuerdos de libre comercio con EEUU y con IVCR $>1$;

c) los aranceles NMF de EEUU son mayores a 0\%, lo que supone que el TTP implicaría su reducción y eventual eliminación y, por tanto, una reducción del precio.

Estas partidas, las cuales se deben identificar bajo la metodología propuesta como las que verían una competencia más intensa en el mercado de EEUU como resultado del TTP, sumaron un total de US\$ 1.47 mil millones de exportaciones medias anuales para el período 2012-2014, equivalentes a un 34\% de las exportaciones de bienes del país a EEUU, un $17 \%$ de las exportaciones totales de bienes, y un $7.5 \%$ de los ingresos corrientes de la Balanza de Pagos.

Cuadro 6. Partidas arancelarias relevantes para la República Dominicana en EL MERCAdo de Estados Unidos EN LAS Que LoS Países DEL TTP TIENEN VCR $>1$ Y ESTÁN SUJETAS A UNA TASA ARANCELARIA MAYOR A $0 \%$

\begin{tabular}{|c|c|c|c|}
\hline $\begin{array}{c}\text { Parti- } \\
\text { da }\end{array}$ & Descripción & $\begin{array}{l}\text { Tarifas } \\
\text { aplicadas }\end{array}$ & $\begin{array}{c}\text { Elasticidad } \\
\text { precio de la } \\
\text { demanda }\end{array}$ \\
\hline 0709 & $\begin{array}{l}\text { Hortalizas, incl. "silvestres", frescas o } \\
\text { refrigeradas }\end{array}$ & $10.4 \%$ & -0.017 \\
\hline 0804 & $\begin{array}{l}\text { Dátiles, higos, piñas “ananás", aguacates "pal- } \\
\text { tas", guayabas, mangos, frescos o secos }\end{array}$ & $4.3 \%$ & -0.004 \\
\hline 2106 & Preparaciones alimenticias, n.c.o.p. & $10.5 \%$ & -0.001 \\
\hline 2401 & $\begin{array}{l}\text { Tabaco en rama o sin elaborar; desperdicios de } \\
\text { tabaco }\end{array}$ & $1.8 \%$ & -0.007 \\
\hline 2402 & $\begin{array}{l}\text { Cigarros "puros", incl. despuntados, cigarritos } \\
\text { "puritos" y cigarrillos }\end{array}$ & $4.2 \%$ & -0.013 \\
\hline 3926 & $\begin{array}{l}\text { Manufacturas de plástico y de las demás materias } \\
\text { de las partidas } 3901 \text { a } 3914\end{array}$ & $2.8 \%$ & -0.028 \\
\hline 6108 & $\begin{array}{l}\text { Combinaciones, enaguas, bragas "bombachas, } \\
\text { calzones" }\end{array}$ & $8.2 \%$ & -0.135 \\
\hline 6109 & T-shirts y camisetas, de punto & $14.5 \%$ & -0.008 \\
\hline 6307 & $\begin{array}{l}\text { Artículos de materia textil, confeccionados, incl. } \\
\text { los patrones para prendas de vestir }\end{array}$ & $3.4 \%$ & -0.01 \\
\hline 6405 & $\begin{array}{l}\text { Calzado con suela de caucho o plástico y parte } \\
\text { superior de materia distinto del caucho }\end{array}$ & $8.1 \%$ & -0.022 \\
\hline
\end{tabular}




\begin{tabular}{rlcc}
6406 & $\begin{array}{l}\text { Partes de calzado, incl. las partes superiores } \\
\text { fijadas a las palmillas distintas de la suela }\end{array}$ & $9.6 \%$ & -0.023 \\
7108 & $\begin{array}{l}\text { Oro, incl. el oro platinado, en bruto, semilabrado } \\
\text { o en polvo }\end{array}$ & $1.2 \%$ & -0.011 \\
$\quad \begin{array}{l}\text { Artículos de joyería y sus partes, de metal pre- } \\
\text { cioso o chapado de metal precioso "plaqué" }\end{array}$ & $6.6 \%$ & -0.011 \\
\hline & Promedio simple & $6.6 \%$ & $-2.2 \%$ \\
\hline
\end{tabular}

Fuente: elaborado con información recuperada de www.trademap.org y Broda \& Weinstein (2006).

\section{Análisis de sensibilidad}

Por último, se requiere estimar el nivel de sensibilidad que podrían tener esas partidas identificadas como sensibles en las exportaciones dominicanas al mercado estadounidense. En principio, habría que considerar dos efectos: el efecto ingreso y el efecto sustitución que la reducción arancelaria provocaría. El efecto ingreso resulta del incremento del ingreso real como consecuencia de la reducción de los aranceles, el cual se traduciría en un incremento de la demanda. El efecto sustitución es el que resulta del cambio en la demanda de un bien por otro, los cuales se asumen como homogéneos, también como consecuencia de la reducción de los aranceles. En este estudio, solo consideramos el efecto sustitución.

La intensidad del efecto sustitución depende de dos factores: el cambio arancelario y la elasticidad precio de la demanda, ambas listadas en el cuadro 6. Como puede observarse, los aranceles más elevados corresponden a confecciones textiles (partidas 6109 y 6108), hortalizas (0709), preparaciones alimenticias (2106), y calzado y sus partes (6405 y 6405). Por su lado, la elasticidad más significativa corresponde a la partida $6108(-0.135)$, siendo el resto significativamente inferior a -0.01 .

Las importaciones estadounidenses totales de esas trece partidas alcanzaron en 2012-2014 un monto medio anual de USD 53.5 mil millones, de las cuales USD 1.38 mil millones fueron originarias de 
países del TTP. Los resultados globales indican que la desgravación arancelaria que el acuerdo supondría incrementaría las importaciones de esos países hasta USD 1.81 mil millones, para un incremento absoluto de USD 432 millones o $31 \%$ respecto al valor medio del período.

El cuadro 7 muestra la distribución del incremento en las importaciones de esas partidas desde todos los países del TTP, esta vez reunidas en grandes grupos de productos. Como se advierte, casi el $88 \%$ del incremento total correspondería a textiles y vestidos, y el $81 \%$ del total del incremento lo explicaría Vietnam. De allí que el 78.1\% del total del incremento de las importaciones desde los países del TTP de los ítems relevantes para República Dominicana sería de textiles y vestidos desde Vietnam. Malasia también aparece como una amenaza en confecciones textiles, además de productos plásticos, explicando un $9.3 \%$ y un $8.6 \%$ del incremento, respectivamente. Otros productos relevantes serían joyas y calzados, pero con una participación en el incremento mucho más reducida ( $1.7 \%$ y $1.5 \%$, respectivamente).

En resumen, las confecciones textiles originarias de Vietnam son, por mucho, la mayor amenaza para las exportaciones dominicanas, seguida también por textiles y vestido desde Malasia, y productos plásticos desde Japón.

Estos resultados apuntan hacia los productos sensibles y el origen de las amenazas y son válidos para cualquier país proveedor, pero no indican los efectos concretos que tendría para las exportaciones de la República Dominicana. Este tipo de estimaciones exceden los objetivos de este trabajo. Pero, al mismo tiempo, los resultados parecen indicar que el valor agregado de un ejercicio como ese, dado el bajo monto de la desviación de demanda (USD 432 millones), es reducido. 
Cuadro 7. Distribución del inCREMENTo de las eXPortaciones de los Países del TTP SIN ACUERDOS DE LIBRE COMERCIO CON EEUU EN PRODUCTOS RELEVANTES PARA LA

República Dominicana, COMO Resultado de la desGravación aRANCELARia

\begin{tabular}{|c|c|c|c|c|c|c|}
\hline Grupos de productos & Brunei & Malasia & Japón & $\begin{array}{l}\text { Nueva } \\
\text { Zelandia }\end{array}$ & $\begin{array}{l}\text { Viet- } \\
\text { nam }\end{array}$ & Total \\
\hline Textiles y vestidos & $0.20 \%$ & $9.3 \%$ & $0.3 \%$ & $0.1 \%$ & $78.1 \%$ & $87.9 \%$ \\
\hline Calzado y sus partes & $0.0 \%$ & $0.0 \%$ & $0.0 \%$ & $0.0 \%$ & $1.5 \%$ & $1.5 \%$ \\
\hline Productos plásticos & $0.0 \%$ & $0.9 \%$ & $6.1 \%$ & $0.5 \%$ & $1.1 \%$ & $8.6 \%$ \\
\hline $\begin{array}{l}\text { Tabaco y productos de } \\
\text { tabaco }\end{array}$ & $0.0 \%$ & $0.0 \%$ & $0.0 \%$ & $0.0 \%$ & $0.0 \%$ & $0.0 \%$ \\
\hline $\begin{array}{l}\text { Alimentos, frescos o } \\
\text { producidos }\end{array}$ & $0.0 \%$ & $0.0 \%$ & $0.2 \%$ & $0.0 \%$ & $0.0 \%$ & $0.2 \%$ \\
\hline Joyas & $0.0 \%$ & $0.0 \%$ & $0.2 \%$ & $1.1 \%$ & $0.3 \%$ & $1.7 \%$ \\
\hline Otros & $0.0 \%$ & $0.0 \%$ & $0.0 \%$ & $0.0 \%$ & $0.0 \%$ & $0.0 \%$ \\
\hline Total & $0.2 \%$ & $10.2 \%$ & $6.8 \%$ & $1.8 \%$ & $81.0 \%$ & $100.0 \%$ \\
\hline
\end{tabular}

Fuente: elaboración propia.

No obstante, está claro que los países más afectados en términos absolutos serían los que mayor participación tienen en las importaciones estadounidenses de esos productos sensibles. En ese sentido, a modo de ilustración, en el caso del capítulo 61 (Prendas y complementos de vestir, de punto), uno de los que mayor peso tiene entre los productos sensibles para la República Dominicana, el 36\% de sus importaciones son originarias de China, el $6.4 \%$ de Indonesia, el $4.9 \%$ de Honduras; mientras que Camboya y El Salvador explican el 4.3\%. En contraste, República Dominicana explica apenas el 0.9\% de las importaciones estadounidenses en ese capítulo.

Esto sugiere que el efecto total del TTP en las exportaciones dominicanas podría ser pequeño, en la medida en que el efecto total se diluiría.

\section{Conclusiones}

Los resultados de este trabajo sugieren que los efectos del TTP en la República Dominicana se concentrarían en 13 partidas arance- 
larias, en las que el peso de las confecciones textiles es muy elevado. Otros grupos de productos sensibles son productos plásticos, joyas y calzados. El principal país beneficiario sería Vietnam y, en menor medida, Malasia. El incremento total de las importaciones sería de USD 438 millones.

La distribución de los efectos entre países y productos se explica, principalmente, por el tamaño de las barreras arancelarias que restringen las exportaciones de esos productos desde esos países asiáticos, así como por la sensibilidad de la demanda a los precios medida por las elasticidades.

A modo de conclusión, los efectos específicos sobre las exportaciones dominicanas parece que serían pequeños porque los efectos globales se diluirán en un alto número de países proveedores de los productos sensibles. En este caso, China podría ser el país más afectado.

\section{Referencias}

Balassa, B. (1965). Trade Liberalization and "Revelead" Comparative Advantage. England: Manchester School.

Broda, C. \& Weinstein, D. (2016). Globalization and the Gains from Variety. Quarterly Journal of Economics, 121(2): 541-585.

Capaldo, J. \& Izurieta, A. (2016). Trading Down: Unemployment, Inequality and Other Risks of the Trans-Pacific Partnership Agreement. GDAE Working paper no. 16-01. Medford, Maryland: Tufts University.

Cheong, Inkyo \& Tongzon, J. (2013). Comparing the Economic Impact of the Trans-Pacific Partnership and the Regional Comprehensive Economic Partnership. Asian Economic Papers, 12(2), 144-64. 
Elliott, K. A. (2016). Not So Free Trade in Textiles and Apparel: Rules of Origin in the TPP. En Assessing the Trans-Pacific Partnership, volume 1. Cimino-Isaacs, C. \& Schott, J. (Eds.). Washington: Peterson Institute for International Economics.

Freund, C., Moran, T. \& Oliver, S. (2016). Tariff Liberalization. En Assessing the TransPacific Partnership, volume 1., PIIE Briefi ng 16-1. Washington: Peterson Institute for International Economics.

Hufbauer, G. C. (2016). Liberalization of Services Trade. En Assessing the Trans-Pacific Partnership, volume 1. Cimino-Isaacs, C. \& Schott, J. (Eds.). Washington: Peterson Institute for International Economics.

International Trade Commission (ITC). (2016). Trans-Pacific Partnership Agreement: Likely Impact on the U.S. Economy and on Specific Industry Sectors. Washington, D. C.

Jackson, J. K. (2016). The Trans-Pacific Partnership (TPP): Analysis of Economic Studies. Congressional Research Service. Washington, D. C.

Lawrence, R. Z. (2016). Studies of TPP: Which Is Credible? Peterson Institute for International Economics. Recuperado de http:// blogs.piie.com/trade/?p=553

Lawrence, R. \& Moran, T. (2016). Impact of TPP on Labor-Market Adjustment and Income Distribution. En Assessing the Trans-Pacific Partnership, volume 1. Cimino-Isaacs, C. \& Schott, J. (Eds.). Washington: Peterson Institute for International Economics.

Petri, P. \& Plummer, M. G. (2016). The Economic Effects of the Trans-Pacific Partnership: New Estimates. Working Paper 
Series 16 -2. Washington: Peterson Institute for International Economics.

Petri, P. A., Plummer, M. \& Zhai, F. (2012). The Trans-Pacific Partnership and Asia-Pacific Integration: A Quantitative Assessment. Policy Analyses in International Economics 98. Washington: Peterson Institute for International Economics.

Strutt, A., Minor, P. \& Rae, A. (2015). A Dynamic Computable General Equilibrium (CGE) Analysis of the Trans-Pacific Partnership Agreement: Potential Impacts on the New Zealand Economy. Preparado por New Zealand Ministry of Foreign Affairs \& Trade, Wellington.

USITC. (2016). Trans-Pacific Partnership Agreement: Likely Impact on the U.S. Economy and on Specific Industry Sectors. May 2016, Publication Number 4607, Investigation Number: TPA105, documento disponible en https://www.usitc.gov/publications/332/pub4607.pdf

World Bank. (2016). Topical issue: Potential Macroeconomic Implications of the Trans-Pacific Partnership. Global Economic Prospect. Chapter 4. January. 